\title{
Norois
}

Environnement, aménagement, société

La Loire. Sociétés, risques, paysages, environnement

\section{L'histoire comparée du Val d'Authion et de la Loire armoricaine en Anjou}

Pour comprendre la nouvelle appropriation citadine des paysages ligériens

History of Val d'Authion and Armorican Loire in Anjou: a comparative approach aimed at understanding new urban appropriations of Loire landscapes

\section{David Montembault}

\section{OpenEdition}

\section{Journals}

Édition électronique

URL : http://journals.openedition.org/norois/880

DOI : $10.4000 /$ norois. 880

ISBN : 978-2-7535-1540-6

ISSN : $1760-8546$

Éditeur

Presses universitaires de Rennes

\section{Édition imprimée}

Date de publication : 1 septembre 2004

Pagination : 47-62

ISBN : 978-2-7535-0054-9

ISSN : 0029-182X

Référence électronique

David Montembault, «L'histoire comparée du Val d'Authion et de la Loire armoricaine en Anjou », Norois [En ligne], 192 | 2004/3, mis en ligne le 20 août 2008, consulté le 01 mai 2019. URL : http:// journals.openedition.org/norois/880 ; DOI : 10.4000/norois.880

Ce document a été généré automatiquement le 1 mai 2019.

(c) Tous droits réservés 


\section{L'histoire comparée du Val d'Authion et de la Loire armoricaine en Anjou}

Pour comprendre la nouvelle appropriation citadine des paysages ligériens

History of Val d'Authion and Armorican Loire in Anjou: a comparative approach aimed at understanding new urban appropriations of Loire landscapes

David Montembault

\section{NOTE DE L'ÉDITEUR}

Cet article a été reçu le 19 avril 2004 et définitivement accepté le 27 juillet 2004.

\section{Introduction}

1 Entre l'Anjou noir, pays des murs de schiste et des toits d'ardoise, et l'Anjou blanc, pays du tuffeau et des habitations troglodytiques, la Loire a creusé son lit, se heurtant à des terrains plus ou moins résistants. Côté est, l'anticlinal constitué de roches tendres du Cénomanien n'a pas résisté aux attaques du fleuve. Les eaux de la Loire, gonflées par la Vienne - aujourd'hui contenues par la levée - ont ouvert un large val dans lequel l'Authion a pu s'engouffrer. Côté ouest, le fleuve n'a pas eu la même facilité à pénétrer les robustes schistes armoricains en partie fracturés. À partir des Ponts-de-Cé, la vallée se resserre et perd donc sa topographie de Val, typique des grandes plaines tourangelles et saumuroises. En l'absence de digues, le fleuve a aussi conservé un tracé hésitant, se divisant fréquemment pour former de nombreuses îles. Au creux des vallées, la Loire a également créé une microtopographie en remaniant ses propres alluvions, formant les montilles (en amont) et les bourrelets de rives, particulièrement favorable aux premiers occupants. 
2 Le fleuve a donc sculpté une armature, c'est-à-dire des constantes paysagères, à l'origine parfois de nombreuses images, souvent poétiques. «Molle et sensuelle contrée!» s'exclamait J. Michelet, «Loire paresseuse » renchérissait $\mathrm{H}$. Taine... Il est vrai qu'en Anjou, les eaux souvent se traînent, serpentant au milieu des sables qu'elles n'ont plus la force de déplacer. Elles peuvent alors sembler oisives...

3 Mais ces traits physiques ne suffisent pas à faire le paysage ni à expliquer les contrastes très forts observés aujourd'hui entre les deux vallées angevines.

4 Côté Bassin parisien, le Val d'Authion, héritier d'un riche passé agricole et autrefois nommé "grande vallée d'Anjou», est composé d'une mosaïque de champs cultivés, parsemée de serres et de quelques prairies bocagères. Ce val n'est plus regardé pour son paysage, remarqué seulement pour la grande levée qui l'isole du fleuve, et qui offre un piédestal aux riches demeures en tuffeau tournées vers les eaux, ou un belvédère aux touristes et aux villageois désirant contempler la Loire.

5 Côté armoricain, la vallée plus sombre et plus étroite est au contraire très observée. Son paysage agraire teinté d'Armorique se fragmente entre de grandes prairies parsemées de frênes et de chênes têtards, des cultures fourragères et des peupleraies. Des constructions héritées du passé racontent aussi la familiarité d'autrefois avec le fleuve : les fermes sur tertre, les épis noyés, les cales et les quais maçonnés sont aujourd'hui restaurés et mis en valeur en souvenir des riches années de l'agriculture, de la pêche et du commerce sur la Loire. À ce titre, ce paysage a été reconnu en 2001 patrimoine mondial de l'Unesco. Mais, pour les amateurs de nature, la vallée conserve aussi un côté "sauvage", fragile, car menacé par la populiculture. Pour "préserver» et «valoriser» ce «paysage remarquable», de multiples mesures ont donc été mises en place depuis dix ans: opérations agri-environnementales, réglementation des boisements, Natura 2000 et zonages multiples de sensibilisation et de classements à la fois écologiques et paysagers... Mais de quelle mise en valeur parle t-on? Les vallées ont toujours été valorisées par leurs riverains et leurs paysages aménagés en conséquence. Et peut-on réellement préserver un paysage et l'isoler de la société qui l'observe?

6 L'histoire comparée des deux vallées angevines nous offre alors quelques clefs de lecture pour mieux comprendre ces paysages ligériens et leur nouvelle perception citadine.

\section{Les leçons du passé}

\section{Permanence d'un système nommé « Paysage »}

7 Quelle définition et quelle(s) valeur(s) donner au paysage? « Les sociétés perçoivent leur environnement en fonction des aménagements qu'elles en font, et elles l'aménagent en fonction de la perception qu'elles en ont» nous dit Augustin Berque (1992). Système complexe, ouvert, vivant et parfois imprévisible, le paysage possède donc au moins deux dimensions qui interagissent pour faire vivre l'ensemble. La première correspond à l'objet concret, hérité des facteurs naturels et de l'action des hommes sur leur environnement. La seconde, plus subjective, est faite d'images perçues. Construites le plus souvent à partir d'une vision utilitaire et sensible du paysage physique, ces images influencent en retour la façon dont les communautés gèrent et aménagent l'objet. Paysage physique et paysage perçu se croisent donc en permanence, se correspondent ou, au contraire, sont en 
décalage. Lorsque l'image rêvée s'éloigne trop de la réalité, le système est en déséquilibre et l'ensemble est susceptible d'évoluer.

Selon cette définition, nous pouvons alors admettre que les anciens fonds agraires étaient déjà des paysages, transformés par les paysans en fonction d'une certaine vision utilitaire des zones inondables. Dans la recherche d'un idéal agraire, ces derniers ont en effet toujours transformé les vallées en ayant une représentation bien précise de ce qu'elles devaient être et de leur rôle pour la communauté. Il s'agissait bien alors «d'images paysagères ", réelles, bien qu'inconscientes.

\section{Évolution des perceptions : une succession d'« images ligériennes »}

9 La première de ces images fut celle de zones à la fois accueillantes et contraignantes pour l'agriculture vivrière. Comme partout en Europe, cette vision est née avec la sédentarisation des premiers agriculteurs - sur les montilles du Val d'Authion - justifiée par la faible mobilité des personnes et l'insuffisance des moyens de transport pour importer ou pour exporter les marchandises. Au-delà d'une simple logique économique, l'histoire montre qu'il s'est agi d'une première "image traditionnelle " forgée, sans doute, à l'époque gallo-romaine et consolidée ensuite au cours du Moyen Âge par l'adoption de principes d'aménagement (calqués sur l'antique système «infield outfield »), par la répétition d'un système de culture (assolement biennal à base de blé, de pois et de fève) et par la mise en place de règles communautaires (vaine pâture sur les prairies et droits communaux).

10 C'est ainsi que, par fidélité à ce modèle, les paysans ligériens ont découpé de façon équitable les terres des bourrelets sableux, perpendiculairement aux cours d'eau (Dion, 1934), en réservant les dépressions latérales aux landes et aux prairies, jusqu'à faire disparaître les derniers bois de vallée, à force de pâturage. Et c'est aussi pour faire durer ce modèle et conserver leur droit de vaine pâture acquis sous Jeanne de Laval ${ }^{1}$ que, contre toute rationalité économique, les habitants du Val d'Authion ont lutté jusqu'à la Révolution pour s'opposer au projet du marquis de Turbilly qui voulait y étendre les cultures textiles. Nul doute alors que, sans la persistance de cette image traditionnelle, la même connaissance du milieu physique aurait pu conduire plus tôt à d'autres paysages...

11 Une seconde image s'est ensuite imposée : celle de vallées fertiles, capables d'alimenter un marché agro-industriel à condition que soit maîtrisée la ressource en eau et que soient développés des moyens de transport. Il a fallu du temps pour que cette vision, née d'un regard extérieur, soit adoptée par l'ensemble des «vallerots». En Anjou, elle ne s'est véritablement imposée qu'après la Révolution avec l'extension des cultures textiles pour la marine à voile grâce au partage des terrains communaux et aux aménagements réalisés pour la navigation. En Touraine, le « jardin de la France », avait connu son heure de gloire dès le XVI ${ }^{e}$ siècle. Par la suite, cette image de vallées fertiles et productives - soit pour l'élevage, soit pour la culture - s'est renforcée, atteignant son apogée au lendemain de la Seconde Guerre mondiale, avec l'adoption de la PAC et de l'ensemble des mesures productivistes.

12 Enfin, en Loire armoricaine, une dernière image agricole est récemment venue effacer les précédentes : celle de zones trop contraignantes et trop peu rentables pour l'agriculture moderne. Pour remplacer la prairie, de multiples encouragements à la populiculture ont alors été mis en place². 
13 Même si l'aménagement agraire des paysages ligériens a longtemps été confié aux seuls travailleurs de la terre, les représentations paysagères leur ont donc souvent échappé. Tous les grands changements observés dans les vallées sont venus de l'extérieur, de la ville principalement. Outre l'influence de grands personnages comme le marquis de Turbilly, le comte de La Bouillerie ${ }^{3}$ ou, plus récemment, le ministre Edgar Pisani, il faut aussi souligner le rôle déterminant des habitants d'Angers pour développer l'activité agro-industrielle au bord de la Loire et de ses affluents : encouragement au retournement des landes et des prairies pour le développement des cultures textiles à partir de la fin du $\mathrm{XVIII}^{\mathrm{e}}$ siècle; encouragement à la populiculture dès la fin des années 1960 ou encore volonté de développer une activité horticole dans le Val d'Authion au cours des années 1970 pour répondre à une demande croissante en plantes ornementales et permettre le déplacement des exploitants du sud d'Angers, menacés par l'extension de la Ville.

La Loire elle-même a été transformée en fonction de différents regards, au prix de nombreux conflits d'usages (Godelaine, 2000). Au fil des siècles, divers aménagements ont ainsi favorisé tour à tour l'agriculture, la pêche, la meunerie ou la navigation. Aujourd'hui, d'autres sont encore prévus, pour rendre la Loire "plus sauvage ${ }^{4}$ ». Mais rien n'indique une véritable rupture. Si ces paysages doivent remplir d'autres fonctions, ils sont toujours aménagés pour ceux qui les côtoient et en partie par eux.

\section{Les paysages ligériens reflet des sociétés riveraines}

15 Malgré des différences très nettes dans l'occupation actuelle des sols, le visage des deux vallées angevines reflète donc, par de nombreux points, les sociétés qui les ont façonnées. Le miroir n'est cependant pas parfait car il faut souvent plus de temps pour qu'un changement s'opère dans le paysage qu'il n'en faut aux idées et aux techniques pour germer et se diffuser - et certaines transformations prévues n'ont jamais eu lieu ${ }^{5}$. De surcroît, le temps se plaît à gommer certaines traces. Mais, dans ces vallées, il subsiste de nombreux indices : des clefs de lecture géographique des paysages.

\section{Les paysages ligériens, reflets de la concentration humaine}

Ancestrales constructions, les paysages ligériens ont subi avant tout les mouvements de population. Est-il alors nécessaire de rappeler que la première cause des déboisements dans les vallées est l'augmentation constante du nombre d'occupants de la fin du Moyen Âge jusqu'à 1850 ? La plus forte concentration humaine dans le Val d'Authion (plus attrayant à cause de sa largeur et de son potentiel agronomique) explique aussi les efforts plus soutenus pour élever la digue et rendre cultivables le maximum de terres. Ensuite, comme ailleurs, l'exode rural entamé dès 1850 , a accompagné la mécanisation permettant de pallier le manque de bras. Dans un deuxième temps, cela a produit l'agrandissement des parcelles et l'intensification des techniques de production pour nourrir des villes en pleine expansion. En Loire armoricaine, cet exode a été si prononcé qu'il a fait craindre les friches et a motivé de nouvelles aides à la populiculture à la fin des années 1980. Enfin, aujourd'hui, suivant la même logique, il est évident que la nouvelle clef de voûte des paysages ligériens tient dans l'échange des populations urbaines et rurales sur les rives de Loire, avec le retour des périurbains dans les villages (fig. 2). 


\section{Reflet d'un mode d'organisation}

17 Certaines transformations observées dans les deux vallées peuvent aussi être interprétées comme le reflet d'un mode de fonctionnement et d'un mode d'organisation des communautés ligériennes. L'embocagement partiel des prairies dans les deux vallées, à la suite de la suppression du droit de vaine pâture au xIX ${ }^{e}$ siècle, illustre bien, par exemple, un esprit individuel lié à un système d'exploitation familial. Certains observateurs ne s'étaient d'ailleurs pas trompés en prévoyant, au lendemain de la Révolution, que les prairies communales seraient beaucoup mieux entretenues en étant partagées plutôt qu'en restant dans l'indivision (le préfet d'Oise en 1810 dans Dion, 1934). Ce même esprit familial explique aussi la division sans limite des terres de vallée jusqu'au lendemain de la Seconde Guerre mondiale dans le souci de léguer à chacun des enfants un patrimoine égal. À l'inverse, depuis le XIx ${ }^{e}$ siècle, sont également venus se superposer dans le paysage des aménagements et des équipements particulièrement uniformes, conçus et réalisés à une échelle dépassant de très loin le périmètre des vallées : les lignes de chemin de fer, les routes et, depuis quelques années, un découpage agraire et des plantations de peupliers très géométriques sans véritable logique avec la micro-topographie (fig. 1). Cette fois, ces éléments illustrent une entreprise collective, intégrant les vallées dans des projets régionaux ou nationaux de développement et d'aménagement.

Figure 1 : Transformation du Val de Louet à la confluence Maine - Loire entre 1958 et 1999 Shifts in land occupation (1958-1999) of the Val de Louet at the Maine - Loire merging.

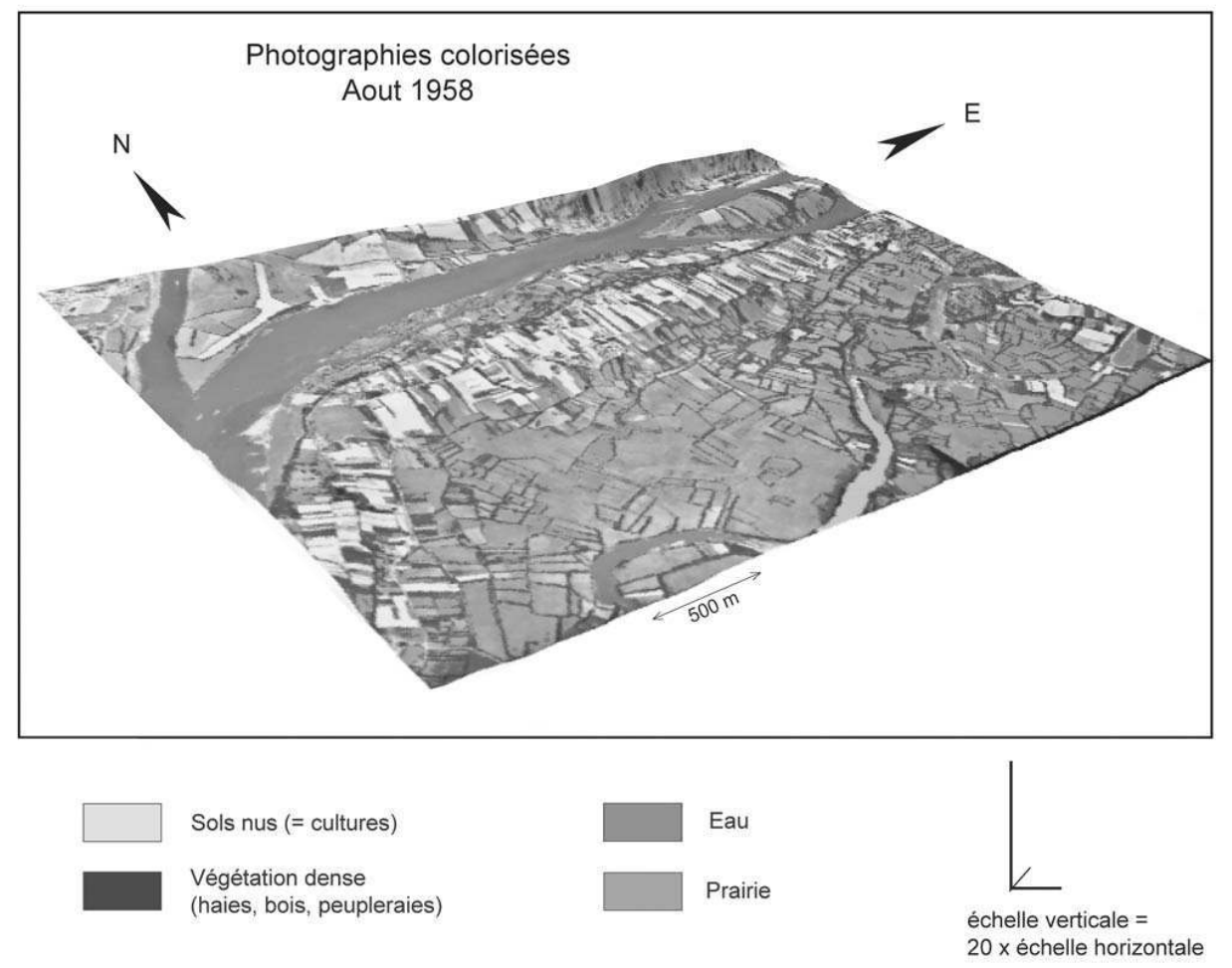

Source : IGN, 1958

D. Montembault, P. Joly Carta 2001 


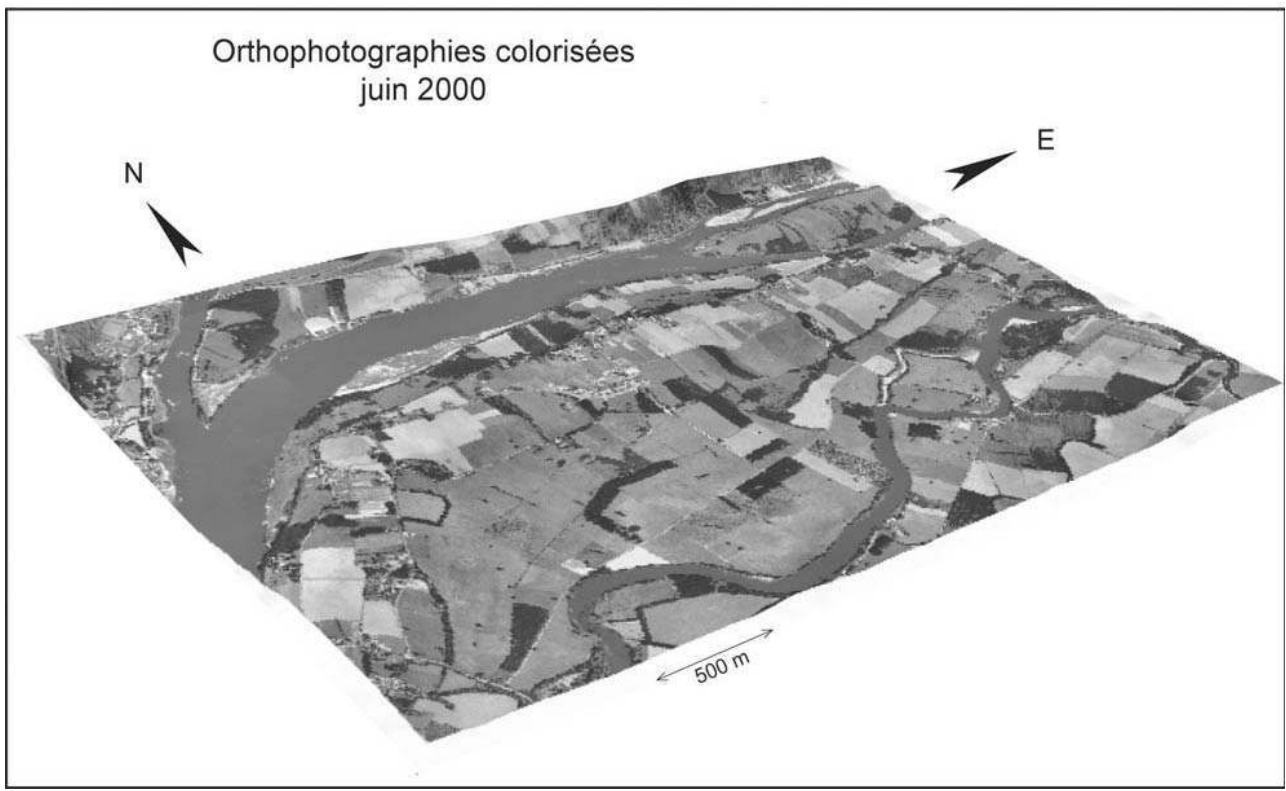

Source : FIT Conseil, 2000

D. Montembault, INH 2004

Figure 2 : Évolution de la population du Maine-et-Loire entre 1851 et 1999 Population evolution (1851-1999) in Maine-et-Loire

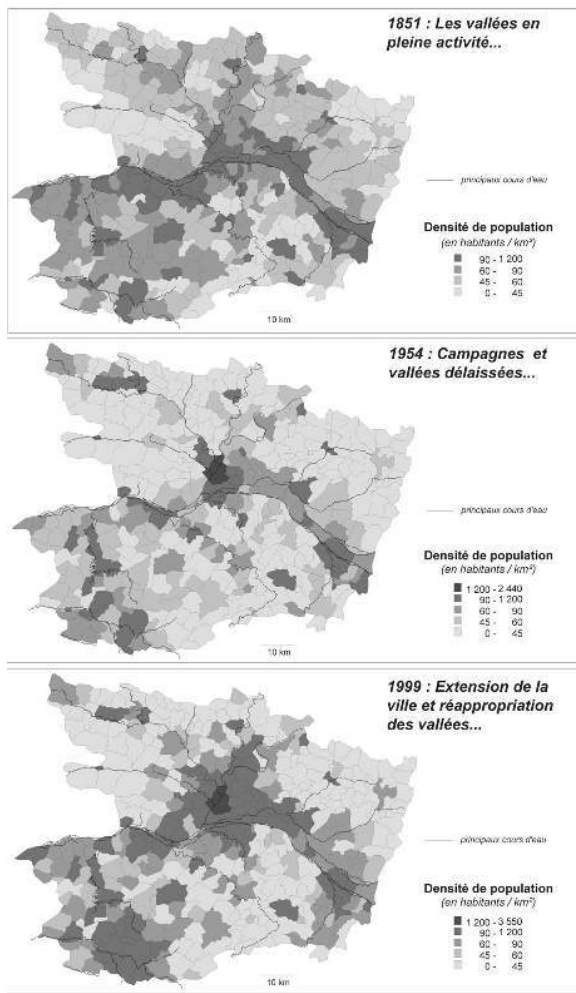

Sources : Dictionnaire d'histoire administrative et démographique du Maine et Loire, INSEE - RGP 1999

D. Montembault, 2002 


\section{Reflet de techniques et d'outils}

Le paysage des vallées a aussi été le reflet d'outils et de techniques qui ont fixé les conditions dans lesquelles ont pu se réaliser les aménagements. Soulignons, par exemple, comment le même désir d'améliorer la production agro-industrielle a conduit, à deux époques différentes, à deux restructurations foncières aux effets totalement inverses sur le bocage. À l'époque du textile, la recherche du meilleur rendement passait par l'ouverture des champs cultivés et par leur extension aux dépens des prés communaux, obligeant souvent à la suppression des haies les plus anciennes. En contrepartie, elle rendait aussi nécessaire la clôture des pâtures pour garder les troupeaux indispensables à la fumure et à la traction animale. Cet embocagement a encore été encouragé par le découpage des communaux en propriétés individuelles, lui-même considéré comme un facteur de progrès. Enfin, la multiplication des arbres (frênes et chênes taillés en têtard), était elle-même nécessaire au bon fonctionnement des exploitations pour le fourrage et le bois que ces derniers apportaient.

Figure 3 : Zonages environnementaux autour d'Angers : les vallées humides « pansements de la ville»

Environmental planning around Angers are humid-valleys acting as "plasters" for the city

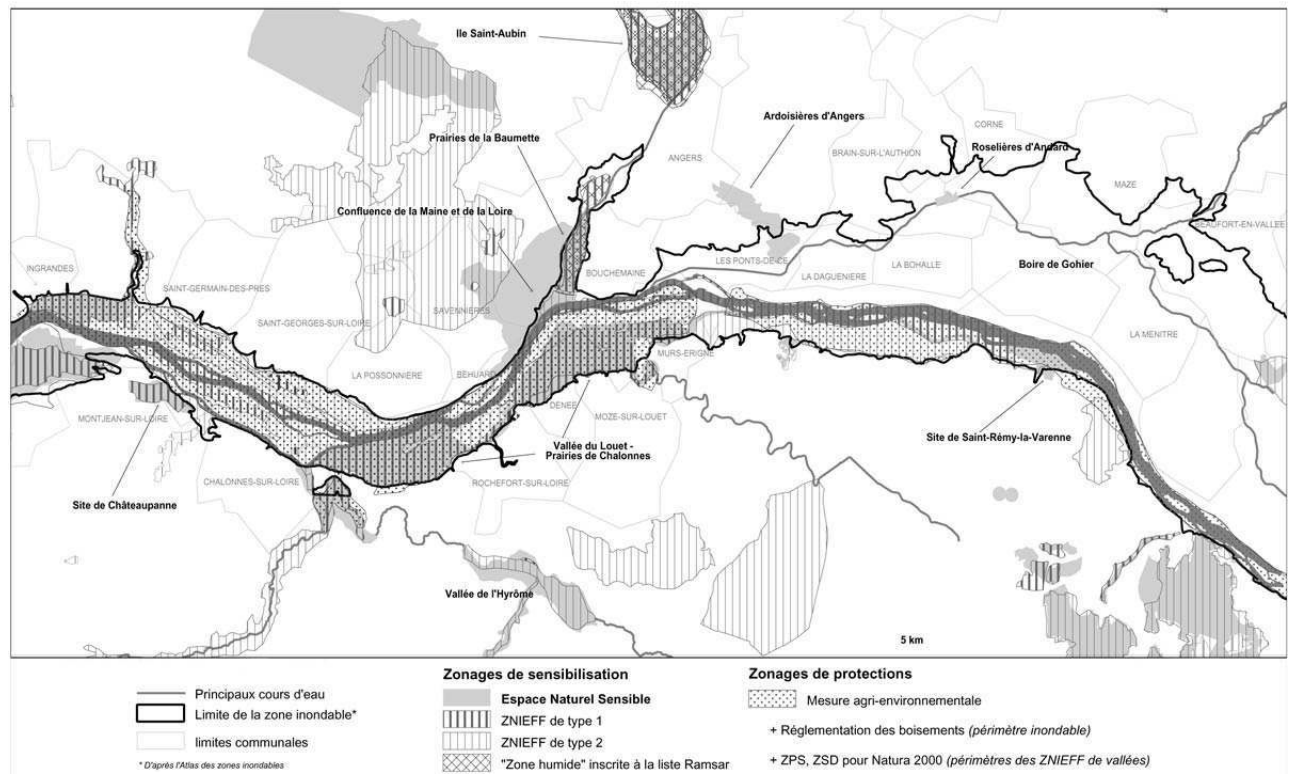

Sources : DDAF 49, ADASEA 49, DDE 49, DIREN Pays de la Loire

David Montembault - INH 2004

Un siècle et demi plus tard, le contexte agronomique ayant été totalement transformé, les haies alors devenues gênantes pour les nouvelles machines ont pu être largement supprimées sans véritable préjudice - selon, bien sûr, les critères de l'époque et la vision agricole dominante des vallées.

Notons que les outils eux-mêmes ont eu un rôle déterminant dans cette évolution: le bocage, planté difficilement à la bêche, a été arraché au bulldozer tandis qu'entre-temps, très peu de progrès avaient été réalisés dans l'outillage pour faciliter l'entretien des haies. À l'inverse, tracteurs, charrues, semoirs et moissonneuses avaient énormément facilité la culture des champs et l'agrandissement des parcelles, encore bêchées à la main au XIX ${ }^{e}$ 
siècle. Aujourd'hui, là où les haies ont retrouvé une nouvelle vocation (écologique surtout), il est frappant de voir apparaittre de nouvelles machines : des broyeurs et des lamiers qui permettent de rétablir un certain équilibre entre le coût d'entretien des haies et celui des autres travaux des champs.

21 Enfin, les outils et les techniques ont également contribué aux images paysagères et à leur diffusion au sein de la plus grande masse. Si, après la Seconde Guerre mondiale, les effets de la PAC ont été si rapides sur le paysage agraire, en comparaison du temps qu'il avait fallu pour faire admettre les cultures textiles, c'est certainement en partie grâce aux échanges accélérés et aux nouveaux moyens de communication utilisés pour faire admettre les changements. Aujourd'hui, le paysage ligérien devient «identitaire »; mais encore faut-il avoir les éléments de comparaison : le « dernier grand fleuve sauvage » l'est au regard des autres fleuves et les vallées angevines « premier site de nidification du Râle des genêts dans la Communauté européenne" le sont en comparaison d'autres zones humides. Les médias et les nouvelles technologies participent donc pour beaucoup à la diffusion des images contemporaines des vallées: la «Loire sauvage» et «le fleuve royal » sont par exemple très présents sur internet, occultant du même coup la Loire agricole $^{6}$.

\section{Reflet d'une vision utilitaire, dominante}

Enfin et surtout, le paysage des vallées s'est inscrit dans son époque en étant un reflet du regard utilitaire posé par l'ensemble d'une collectivité sur son territoire, niant ainsi les perceptions plus individuelles, et peut-être plus sensibles, sur des espaces réduits. Ces visions dominantes n'ont pas seulement concerné directement le paysage mais également les outils, les techniques, les normes, les lois et les règlements dont le premier a bénéficié. C'est donc ce qui rend particulièrement complexe le système et son étude et qui explique parfois son inertie.

Les mesures actuelles visant à préserver les paysages et leur richesse écologique (en limitant la plantation de peupliers dans les vallées par exemple) n'auraient jamais autorisé les cultures textiles au XIX ${ }^{\mathrm{e}}$ siècle (très polluantes) qui ont pourtant fait toute la richesse des « vallerots » et de leurs constructions paysagères (fig. 4). 
Figure 4 : Le paysage du Val d'Authion The Val d'Authion landscape

Le paysage du Val d'Authion vu d'avion et le paysage du Val d'Authion vu par l'observatoire de la Vallée d'Anjou
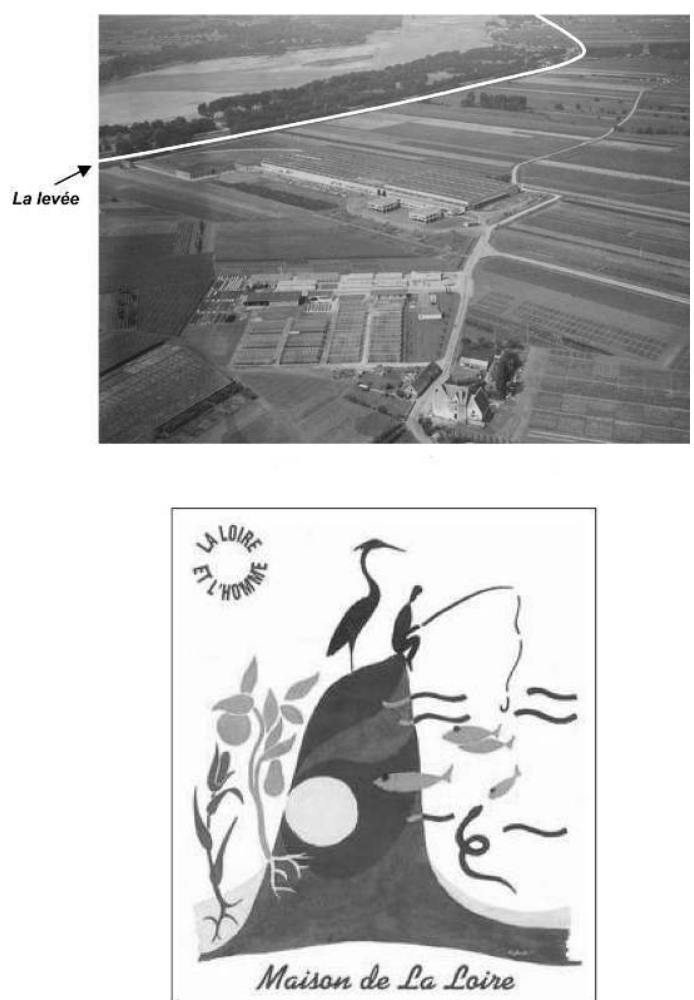

Ce regard, tourné vers le fleuve, exprime bien les liens entre l'homme et le paysage (avec la levée) et entre la Loire et sa vallée fertile. Cependant, le logo très stylisé offre une vision excessivement

bucolique de ce système paysager, très axé sur le tourisme et l'écologie.

\section{Le paysage ligérien, résultat d'actions plus que somme de représentations}

Avant de s'intéresser à la demande paysagère actuelle et à l'avenir possible des vallées ligériennes, il nous faut encore insister sur un dernier point, à savoir la prédominance des faits sur les représentations.

En effet, si le paysage physique s'inscrit autant dans une société et une époque, trahissant les habitudes de ceux qui le font vivre, c'est surtout parce que l'objet est sensible à tout un ensemble d'actions, souvent absentes des représentations. Le paysage vécu ne peut pas être totalement isolé de tous les événements qui se déroulent sur les autres parties du territoire. L'histoire montre ainsi qu'il ne suffit pas de "penser le paysage " pour que l'objet physique évolue vers une image rêvée et que, à l'inverse, des actions non perçues comme transformatrices de paysages peuvent laisser une empreinte très forte sur le territoire.

$\mathrm{Au} \mathrm{XIX}^{\mathrm{e}}$ siècle, par exemple, malgré l'émergence du mouvement romantique et la multiplication des représentations poétiques de la Loire et de sa vallée, le paysage physique n'a pas été transformé dans un but esthétique pour faire naître de nouvelles émotions. Au contraire, en réponse à une demande nationale, il a été totalement aménagé 
pour produire en plus grande quantité des fibres textiles. La raison économique a donc pris le pas sur la vision poétique, sans pour autant lui nuire... Aujourd'hui, les images poétiques de la Loire continuent à se nourrir du paysage physique et intègrent parfaitement les aménagements rendus possibles grâce à la vente des biens communaux et au succès du chanvre : les riches demeures en tuffeau, les ponts en fer (si décriés par Flaubert ${ }^{7}$ ), les cales et les quais refaits au XIx ${ }^{e}$ siècle ont participé pour beaucoup au classement de la Loire au titre de paysage culturel de l'Unesco.

Aujourd'hui encore, ce n'est pas parce que certains "motifs caractéristiques" sont conservés dans les vallées que le paysage tout entier reste inchangé. Derrière les frênes têtards, il manque tout ce qui participait à la vie du bocage: sur les sentiers de découverte, les joggeurs et les promeneurs ne créent pas la même " ambiance paysagère " que les lavandières ou les enfants en culotte courtes; les signes de couleur qui sont destinés aux premiers n'auraient pas beaucoup de sens aux yeux des seconds. Au sein des villages restaurés, les boutiques mises aux normes n'ont pas gardé l'aspect premier des vieilles échoppes, les nouvelles routes ont très peu de points communs avec les anciens chemins ruraux peu carrossables. Enfin, les bruits (jusqu'à 110 trains par jour entre Angers et Chalonnes) et les odeurs qui parcourent les vallées ne sont pas non plus ceux d'autrefois... Bref, les paysages restaurés de la Loire comportent en vérité une multitude de signes qui les font appartenir au présent. Malgré une image traditionnelle que certains voudraient conserver, le paysage, territoire vécu, continue donc d'évoluer.

\section{Quels paysages de vallées pour demain?}

\section{Le " triomphe de l'urbanité » fait apparaître une nouvelle demande pour les paysages ligériens}

Même si la période des grands bouleversements paysagers semble révolue, il faut donc encore s'attendre à de nouveaux changements dans les vallées ligériennes, par petites touches sensibles, en réponse à de nouvelles attentes et à de nouvelles perceptions. Nous l'avons déjà évoqué, rien n'indique une véritable rupture dans la façon de gérer ces zones inondables. Si elles doivent répondre à des besoins plus actuels, elles restent toujours un espace de projets pour les communautés riveraines. Cependant, le « système paysage » est aujourd'hui beaucoup plus complexe. Les acteurs sont plus nombreux et surtout plus diversifiés. Les échelles ont éclaté: le marché des produits agro-forestiers s'est mondialisé, les politiques d'aménagement tout comme les normes de production sont couramment dictées par l'Union Européenne et le regard porté sur les vallées, nourri d'images de campagnes riantes et de nature généreuse, relayées par les médias, est luimême fortement influencé par une culture citadine qui s'uniformise à l'échelle du pays.

S'il y a eu fracture alors, ce n'est pas à proprement parler au niveau du paysage mais plutôt au cœur de la société. La civilisation paysanne qui, depuis plusieurs millénaires avait participé à la construction des paysages agraires, a été balayée en quelques décennies et, depuis 1850 , la presque totalité des enfants des travailleurs de la terre a rejoint des carrières urbaines, désertant en premier lieu les terroirs trop contraignants comme les vallées inondables.

30 Aujourd'hui, dans la grande couronne d'Angers, les bourgs et les villages se repeuplent (fig. 2). Mais, sur le plan des valeurs et du mode de vie, cette migration correspond plus à 
une extension de la ville qu'à un véritable retour à la campagne. Les urbains quittent l'agglomération, au sens premier du terme, pour habiter des lotissements dans les villages ou pour restaurer quelques vieilles fermes en y installant tout le confort moderne et, dans la mesure de leurs moyens, en y conservant le charme ancien. «L'urbanité triomphe hors de la ville» (Hervieu et Viard, 2001) et, avec elle, émerge un nouveau regard dominant sur le paysage rural et sur les vallées en particulier. Ce changement se traduit par deux types de demandes : l'une, consciente, née semble-t-il, à la fois d'un mode d'appropriation du paysage par le regard et par des besoins issus de la vie citadine et l'autre, inconsciente, économique, liée aux habitudes de confort et de consommation.

\section{Une demande consciente pour le paysage visible : à la fois très nostalgique et très actuelle}

31 La ré-appropriation des vallées angevines par les urbains leur offre donc un nouveau droit de regard sur ces paysages. Mais les liens directs avec la terre et le fleuve ayant été supprimés, la demande consciente de paysage se limite essentiellement à sa dimension visible et consommable dans le cadre d'activités de loisirs. La majorité des acteurs, excessivement mobiles, ne sont plus que de passage dans les vallées, à des moments choisis, et leurs « images paysagères » nées de la contemplation se nourrissent beaucoup de nostalgie (agraire et marinière), s'inspirant aussi fortement des images des peintres et des poètes des siècles passés, dont les œuvres sont abondamment exhumées (Montembault, 2002). Avec la montée en puissance du mouvement écologiste, la nature aussi fait partie du spectacle; grossies à la loupe ou à la jumelle, faune et flore sont les pièces précieuses du musée ligérien (à ne toucher qu'avec les yeux).

En quelque sorte, au "théâtre des champs ${ }^{8}$ ", les acteurs ont déserté la scène et admirent le décor. Chacun, simple spectateur ou metteur en scène, plus ou moins confortablement installé dans son nouveau rôle, observe, applaudit ou fait de mauvaises critiques. Entre deux séances d'observation, quelques hommes doivent lutter contre les attaques du temps et faire en sorte que ce décor vivant conserve tout son éclat. Mais, le travail est peu valorisé, confié à des jeunes chômeurs, des RMIstes ${ }^{9}$ ou bien encore à des agriculteurs, en échange de quelques primes...

Certes, ce tableau est caricatural. Mais, il permet de saisir la nouvelle demande paysagère, très exigeante, et parfois sans réalité avec les contraintes physiques et économiques qui ne sont plus directement vécues.

\section{Un désir d'enracinement}

Une première attente pourrait s'interpréter comme un désir d'enracinement. Elle se traduit par la volonté de sauvegarder quelques éléments visibles du paysage, hérités de la paysannerie et de la navigation (en Basse-Loire surtout). Légèrement épargnées par les Trente Glorieuses, les zones inondables ont, en effet, conservé un patrimoine plus riche qu'ailleurs. Au milieu de paysages de plus en plus standardisés, les frênes têtards, les prairies avec leurs boires, les habitats sur tertre associant le schiste et le tuffeau, les quais de Loire maçonnés en pierre, deviennent alors les attaches nouvelles d'une population en manque de repère, « en perpétuelle oscillation entre un local sans enracinement possible et un global mal maîtrisé » (Humeau, 1975). 

l'ensemble des urbains en réaction à l'effacement des cultures provinciales. Ce fut la grande période des études folkloriques (1879-1914); «on étudia les paysans comme une espèce en voie de disparition » nous rappelle $\mathrm{E}$. Weber (1983). Aujourd'hui, dans un autre courant passéiste, la nostalgie se reporte sur le paysage, dernier élément visible d'une culture disparue, dernière attache pour une civilisation aux racines paysannes, ultime vestige $d u$ « pays » en quelque sorte.

\section{Une demande identitaire}

Une autre demande, qui peut se rattacher à la précédente, concerne le paysage «identitaire». Face à une certaine uniformisation de la culture et des modes de vie, il revient aux paysages ligériens de marquer la différence. Cette attente se traduit, encore une fois, par la conservation de motifs paysagers traditionnels, en insistant cette fois sur ce qui fait leur caractère typique ou pittoresque, en mettant en avant ce que les autres ne possèdent pas. En Loire armoricaine, les grandes prairies associées au frêne têtard, le chanvre et le lin disparus au début du siècle, ont fait oublier le blé cultivé depuis le Moyen Âge, ainsi que le chou, la betterave fourragère, les haricots verts ou encore le tabac qui, tour à tour, ont fait la richesse des cultivateurs ligériens au cours du xxe siècle.

La demande de paysage identitaire ne correspond donc pas à un simple désir de mémoire mais plutôt à l'envie de conserver quelques éléments typiques à partir desquels il devient possible de se réinventer un passé, idéalisé, folklorisé, unique. On assiste, en quelque sorte, à une restauration du paysage par le regard, par la projection de valeurs contemporaines, sur des éléments hérités du passé. comme une création écologique servant à diriger la circulation des eaux ${ }^{10}$ (Mission bocage, 1997). Si cela ne correspond pas à la fonction première des haies, démontrée par R. Dion ou M. Bloch ${ }^{11}$, cela reflète cependant une demande très actuelle d'amélioration de l'environnement.

De même, dans le Val d'Authion, "vingt ans ont suffi pour gommer l'identité du pays de la carotte et de l'oignon » constate le maire de Mazé, soulignant que, lors du dernier passage du Tour de France dans sa commune, un journaliste en faisait un savoureux portrait en proclamant : «À Mazé, le champion, c'est l'oignon!» Aujourd'hui, ce même paysage agraire n'est plus perçu de la même manière (fig. 4) et certains néo-ruraux, à la recherche du calme de la campagne, se plaignent du bruit nocturne des systèmes d'arrosage (Delépine, 2001).

Les exemples sont encore nombreux, de multiples communes cherchant à se greffer une nouvelle identité sur leur patrimoine paysager, souvent à l'aide de panneaux d'information centrés sur le tourisme et la consommation de nature.

Dans cette double quête d'identité et d'enracinement, la face la plus regardée des vallées (celle visible depuis la route et les coteaux ou depuis les aires d'accueil «paysagées ») est alors devenue très surveillée ${ }^{12}$. C'est à elle que revient la lourde responsabilité de créer des points de repère et d'instaurer une différence entre des populations de plus en plus imprégnées par la même culture et habituées au même mode de vie. 


\section{Une demande d'environnement} plus nombreuses, sinon plus quotidiennes et plus matérielles, devenues semble-t-i inconscientes mais tout aussi capables (sinon plus?) de faire évoluer les paysages ligériens. Elles sont essentiellement d'ordre économique, liées aux règles de production, aux habitudes de consommation et à des normes de confort devenues incontournables. À un niveau local, citons, par exemple, l'amélioration des chemins vicinaux et la construction de parkings à proximité des zones de loisirs, l'installation du tout-à-l'égout et de l'éclairage public dans les villages, la construction de centres culturels, de centres sportifs et même, de lotissements en zone inondable comme aux Ponts-de-Cé. La liste est longue. Et toutes les communes ligériennes devenues les dortoirs d'Angers, sont déjà confrontées à ces nouvelles demandes de services, très transformatrices pour leur paysage et parfois peu compatibles avec leur budget ${ }^{13}$. Enfin, à ces multiples besoins gérés à des échelles variées, s'ajoute aussi toute une série de logiques : logique de rendement, de spécialisation et d'efficacité à tout prix, logique de gestion administrative du territoire et logique de consommation... Les mêmes qui ont conduit au cours des Trente Glorieuses à la transformation des « paysages paysans ».

\section{Des demandes paysagères paradoxales?}

Il pourrait alors apparaître un certain paradoxe entre la demande consciente de paysage et la demande inconsciente, entre la volonté affichée de retrouver des paysages d'antan en partie idéalisés et le manque de volonté de remettre en cause la civilisation qui les a fait disparaître. Comment en effet regretter les " paysages paysans ", tout en plébiscitant des produits toujours moins chers, des règles d'hygiène et de sécurité toujours plus strictes, des délais de production et de fourniture toujours plus réduits? Comment refuser une centrale nucléaire dans le paysage ligérien ou pourquoi pas, des éoliennes, tout en consommant toujours plus d'électricité ${ }^{14}$ ? Comment enfin accuser une peupleraie

Norois, 192 | 2008 
de défigurer son cadre de vie tout en augmentant sa consommation d'emballages issus du déroulage $e^{15}$ ?

Ce paradoxe est vite levé en vérité à travers deux logiques paysagères, déjà largement répandues et, semble-t-il, encore appelées à se développer : d'une part, celle du zonage du territoire en multiples aires, zones de production spécialisées et, d'autre part, celle de «l'habillage» du paysage permettant d'en renvoyer une image désirée grâce à des façades évocatrices.

\section{Un découpage de plus en plus poussé}

Le zonage à toutes les échelles apparaît donc comme la solution la plus évidente, capable de lever la contradiction entre un désir de paysages traditionnels et des actions qui tendent à les effacer. Le morcellement de l'espace a déjà conduit à la séparation des deux vallées angevines en deux paysages agraires bien distincts, par rationalité économique et compte tenu surtout des conditions physiques inégales. Or, si les premiers découpages ont vu le jour sans véritable volonté, aujourd'hui à l'inverse, le zonage de l'espace est devenu une vraie logique de gestion, permettant à la fois de mieux cibler les actions et de répartir plus facilement les subventions. Cette politique menée à toutes les échelles, est d'ailleurs à l'origine d'une quantité impressionnante de sigles dont la première lettre, toujours commune, signifie la zone. Après les ZUP, les ZAD et les ZAC en périphérie des villes, voici donc les ZPPAUP pour isoler les paysages remarquables ; les ZNIEFF, les ZICO, les ZSC, et les ZPS pour délimiter les zones naturelles sensibles; les Zones libres au boisement, les Zones interdites au boisement et les Zones soumises à autorisation, pour maitriser la plantation des peupleraies dans les vallées.

\section{L'habillage du paysage}

L'autre façon de lever la contradiction d'une image voulue peu compatible avec le vécu consiste à habiller les vallées en soignant particulièrement leur partie visible. C'est, par exemple, l'installation d'un «bocage horticole» voulu par la chambre d'Agriculture, devant servir à camoufler les serres du Val d'Authion (Lagel et al., 2000) ou de manière plus générale, l'embellissement des zones d'accueil et des circuits de randonnée dans les vallées, la dissimulation des éléments jugés dégradants comme les stations d'épuration, les châteaux d'eau, les stabulations agricoles, etc., tous masqués derrière des façades plus flatteuses pour le regard et plus en accord avec les attentes du public. Faute d'assumer nos équipements modernes, le paysage ne doit donc plus renvoyer la simple image de ce qu'il est, mais donner l'image de ce que nous voudrions qu'il soit. Il doit évoquer, signifier, ne plus être neutre.

Dans cette double logique de découpage de l'espace et d'habillage du paysage, on risque donc d'assister à un morcellement de plus en plus poussé des vallées avec la juxtaposition d'une multitude de paysages à la fois très spécialisés et très productifs : paysages écologiques, paysages touristiques, paysages de vente à la ferme... Les bords de Loire seront peut-être découpés en multiples zones très spécialisées, elles-mêmes aménagées avec une façade visible (correspondant à l'échelle du regard), avec une zone de production, moins soignée sur le plan esthétique; des paysages en plusieurs couches, adaptés à la fois à l'exigence du regard et aux exigences de production à une échelle plus vaste. 


\section{Conclusion}

Dans notre société citadine, les vallées encore inondables, autrement appelées zones humides, comme la Loire armoricaine, ont donc un nouveau rôle à jouer. Dans le découpage européen de l'espace agricole, c'est à elles qu'ont été confiées en priorité les fonctions d'épuration des eaux; de conservation des espèces faunistiques et floristiques ; d'expansion des crues devant protéger les populations urbaines. Dans le découpage de la France touristique, les paysages ligériens nouvellement entrés dans le patrimoine mondial de l'Unesco, trouvent aussi une place de choix, ils sont censés attirer un nombre de visiteurs toujours croissant. À l'échelle du département enfin, les communes de vallées concentrent une nouvelle population à la recherche d'un cadre de vie agréable, en quête de paysages à la fois beaux, sains et traditionnels.

51 Si la ré-appropriation des vallées par le regard des citadins laisse penser que beaucoup d'efforts seront réalisés pour embellir leur face visible, des interrogations demeurent quant à l'avenir des paysages dans leur entier, en particulier du côté de leur face cachée.

Qui va entretenir les vallées? Le paysage rural ne peut pas être un théâtre sans acteurs, or aujourd'hui, le nombre d'agriculteurs dans les zones inondables est en constante diminution. Si la majorité des néo-ruraux se sentent donc aujourd'hui spectateurs du paysage rural, ils oublient qu'en réalité, ils en sont les principaux acteurs et que l'avenir de ces paysages dépend beaucoup de leur participation directe ou indirecte (financière).

53 Une deuxième série de questions s'impose : Comment va réagir la Loire? Quelle réaction aura l'hydrosystème face au rehaussement de la ligne d'eau? Les espèces vont-elles s'adapter et réintégrer les habitats restaurés en leur nom, ou vont-elles continuer à déserter, à la recherche de conditions plus favorables, ou bien encore disparaître sans solution de repli ? ligériens...

\section{BIBLIOGRAPHIE}

BERQUE (A.), 1992. - « La représentation des paysages - Une certaine conception de l'environnement », dans Brunet (P.) (dir.), Atlas des paysages ruraux de France, Paris, Éd. J.-P. Monza, 190 p., p. 108-121.

DelePine (S.), 2001. - Étude sur la commune de La Ménitré, rapport d'enquête, département de Géographie, Université d'Angers, 20 p.

Dion (R.), 1934. - Le val de Loire - Étude de géographie régionale, Tours, Arrault et C $\mathrm{C}^{\mathrm{ie}}, 710 \mathrm{p}$.

-, 1981. - Essai sur la formation du paysage rural français, Paris, Flammarion, $173 \mathrm{p}$.

Dubreuil (J.), 1942. - Économie rurale de l'Anjou, Angers, Imp. de l'Anjou, 247 p. 
GODELAINE (F.), 2000. - « Les conditions de navigation sur la Loire et la Mayenne de la fin du XVII ${ }^{\mathrm{e}}$ siècle à la Révolution ", Archives d'Anjou, n 4, p. 47-62.

HERVIEU (B.), VIARD (J.), 2001. - Au bonheur des campagnes, La Tour-d'Aigues, Éditions de l'Aube, coll. « Monde en cours », $150 \mathrm{p}$.

HUMEAU (J.-B.), 1975. - La production maraîchère et légumière dans la vallée de l'Authion, thèse de $3^{\mathrm{e}}$ cycle, Université de Caen, $468 \mathrm{p}$.

LAGEL (D.) et al, 2000. - « La vallée de l'Authion. Amorcer la reconstruction d'un paysage déstructuré ", dans Agriculture et paysage, rapport d'étude de la chambre d'Agriculture du Maineet-Loire, p. 107-187.

1997. - La Loire et son bocage, rapport de la Mission Bocage et du Conservatoire régional des rives de Loire et de ses affluents, $21 \mathrm{p}$.

Montembault (D.), 2002. - Les vallées face à l'appropriation urbaine - Des mutations de l'occupation du sol dans les vallées proches d'Angers aux nouveaux paysages, Thèse de doctorat, Université d'Angers, $409 \mathrm{p}$.

SCHUle (C.-A.), 1996. - «L'eau et les paysages dans l'espace urbain et périurbain d'Angers », Hommes et Terres du Nord, p. 49-55.

1975. - Vallée du Louet. Protection contre les crues de printemps. Étude de l'intérêt économique agricole, rapport de la chambre d'Agriculture du Maine-et-Loire, $24 \mathrm{p}$.

WEBER (E.), 1983. - La fin des terroirs. La modernisation de la France rurale 1870-1914, Paris, Fayard, 839 p.

\section{NOTES}

1. Selon un édit de 1471 qui ne faisait que confirmer des pratiques déjà ancestrales.

2. La production a été vivement encouragée dès la période 1966-1968 (Edgard Pisani était alors ministre de l'Agriculture, puis fut député du Maine-et-Loire), avant d'être favorisée par de nombreuses aides (exonérations fiscales, aides FFN, contrats de plan, etc.)

3. Fondateur du premier « Syndicat Agricole de l'Anjou » en 1887.

4. Creusement de boires, construction du seuil d'Ingrandes par exemple.

5. Comme le projet d'assainissement de 2000 ha de terres inondables entre Les Ponts-de-Cé et Montjean prévu en 1954 et ré-étudié très favorablement en 1975 (C.A. du M.L. 1975).

6. À titre d'exemple, une recherche avec le moteur Yahoo en décembre 2000 avait donné le résultat suivant: 59 références pour l'expression «Loire sauvage» plus un site: [http:// www.loire-sauvage.com], contre seulement 13 pour «Loire navigable » et aucune pour «Loire agricole ».

7. Voici ce qu'écrivait Flaubert, à la vue du pont d'Ancenis : « Rien ne m'attriste et ne me donne pire humeur qu'un pont suspendu avec son tablier aérien et l'eau qui passe sans obstacle sous lui [...] Il est possible que ce soit un progrès de l'art et une utilité pour les grands voiliers ; mais c'est mortel pour le paysage, et, ma foi, la nature vaut bien quelquefois la peine qu'on se gêne pour elle. »

8. En 1600, Olivier de Serres publiait le premier traité d'agronomie sous le titre "Théâtre d'agriculture et message des champs $»$.

9. Selon le Conservatoire régional des rives de la Loire et de ses affluents, entre 1994 et 2002, 29 chantiers de réinsertion organisés par le conservatoire ont ainsi employé plus de 320 personnes (RMIstes, chômeurs) sur le Maine-et-Loire et la Loire-Atlantique. 
10. «Un lien spécifique unit le fleuve et le bocage: les haies ont été très souvent plantées perpendiculairement à l'écoulement des crues dans un double souci de préservation contre l'érosion des sols et d'enrichissement des terres par sédimentation des limons transportés par la Loire » affirme la Mission Bocage.

11. Il semble en effet établi que la création du bocage a été une initiative privée, postérieure au partage cadastral : la haie n'a donc pas servi à découper, elle est venue marquer des limites déjà établies, mis en servant avant tout de clôture pour les animaux et les propriétés.

12. Cf. CRAPE de la Corniche Angevine.

13. À Mazé, par exemple, dans le Val d'Authion, le maire déclare : «Dites à quelqu'un de la ville d'avoir son système d'assainissement autonome : pas question. Il veut se brancher sur le tout-àl'égout [...]. Les chemins sont devenus des rues à part entière [...], la moitié du kilométrage est passée du stade de chemin rural à celui de voie urbaine. Enfin, certains nouveaux arrivants n'hésitent pas à réclamer l'éclairage public à 3 ou 4 kilomètres du bourg. "

14. Progression de la consommation intérieure d'électricité française de 3,9\% entre 2002 et 2003 (source : EDF).

15. En l'an 2000, en France, le bois de peuplier est entré dans la production de 480 millions de caissettes de fruits et légumes, 30 millions de boîtes de fromages, 12 millions de bourriches de fruits de mers, des millions de supports de pizza, bâtons de glaces, etc. (données INSEE).

\section{RÉSUMÉS}

À la frontière du Bassin parisien et du Massif armoricain, l'Anjou possède deux vallées ligériennes aux visages bien différents. Côté est, le Val d'Authion, protégé du fleuve par une grande levée, continuellement transformé depuis des siècles pour l'agriculture intensive, est aujourd'hui peu valorisé pour son paysage. Côté armoricain au contraire, la vallée inondable bénéficie de nombreuses mesures de gestion, visant à la fois à "préserver et valoriser " un patrimoine naturel et culturel de plus en plus reconnu. Mais peut-on véritablement protéger un paysage, sans tenir compte de l'ensemble des facteurs qui ont participé à sa genèse? Inversement, la nouvelle perception citadine des paysages ligériens ne conduit-elle pas à de nouvelles transformations? L'histoire comparée de ces deux vallées angevines offre quelques clefs de lecture pour, d'une part, mieux saisir la demande paysagère actuelle et, d'autre part, prévoir quelques évolutions futures.

At the border of the Bassin parisien and the Massif Armoricain, the Anjou region contains two very different valleys. On the east, the Val d'Authion, protected from the Loire River by a high and continuous levee constantly transformed for centuries for intensive agriculture, is currently little appreciated for its landscapes. On the armorican side, on the contrary, the valley, still floodable, benefits from numerous management projects aiming at "preserving and improving" a natural and cultural heritage increasingly recognized. But can a landscape be really protected without taking into account all the factors that have been involved in its genesis? Conversely, doesn't the new urban perception of the Loire landscapes - the "Ligerian" landscapes - lead to new transformations? A comparative historical analysis of these two Anjou valleys reveals a few clues for a better understanding of the current demand for landscapes and a better prediction of future developments. 
INDEX

Mots-clés : appropriation de l'espace, paysage rural, vallée, zone humide

Keywords : appropriation of land, rural landscape, valley, wet zone

Index géographique : France, Loire (fleuve et vallées), Authion (rivière et vallées), Anjou, Maineet-Loire

\section{AUTEUR}

\section{DAVID MONTEMBAULT}

département paysage - Institut national d'Horticulture (Angers), david.montembault@inh.fr 